\title{
Incremental Robot Skill Learning by Human Motion Retargetting and Physical Human Guidance
}

\author{
Dongheui Lee ${ }^{1}$ \\ ${ }^{1}$ Chair of Automatic Control Engineering, Department of Electrical and Computer Engineering \\ Technical University of Munich (TUM), 80290 Munich, Germany (E-mail: dhlee@ tum.de)
}

\begin{abstract}
Research on skill acquisition and generalization to a different scenario has grown steadily in importance and became now a main topic of robotics research. Imitation learning, one of the main streams for robot learning, provides an efficient way to learn new skills through human guidance, which can reduce time and cost to program the robot. This extended abstract presents our research on incremental skill learning through physical human robot interactions. We introduce our method to teach a robot how to learn synchronized and coordinated whole body motions. Our controller provides a human user comfortable assistance for physical guidance beyond the gravity compensation. External force torque estimation allows further possibilities. One is teaching motion primitives of a legged humanoid robot by taking human intervention into consideration for a balancing problem. Another is teaching multiple tasks like end-effector motions and null space motions. The proposed algorithms are verified on multiple robotic systems including full size humanoid robots.
\end{abstract}

Keywords - Incremental skill learning and refinement, physical human robot interaction.

\section{Introduction}

Research on robot learning from demonstration has received great attention in the last decade since it can serve a useful methodology for intuitive robot programming, even by general users without robotics expertise [6][2]. In the earlier works of this field, demonstrations were provided either by teleoperating the robot or by vision/motion sensors recordings of the user doing the task. In the case of using human motion data directly, we need to solve the motion retargetting problem from a human user to a target robot. Kinematics and dynamics differences between the demonstrator and the robot must be handled and it is very hard to ensure an optimal retargeted motion. Thus it may be desired that a human supervisor can modify the shape and timing of retargeted motion primitives in an intuitive way.

The recent hardware and software developments towards compliant and tactile robots can offer the user to physically interact with the robot to transfer or refine skills. Physical interaction in the context of robot learning is a young but promising upcoming research topic (see Fig. 1). It provides a natural interface to kinesthetic

This paper is partly supported by the European Community within the FP7 ICT-287513 SAPHARI project.

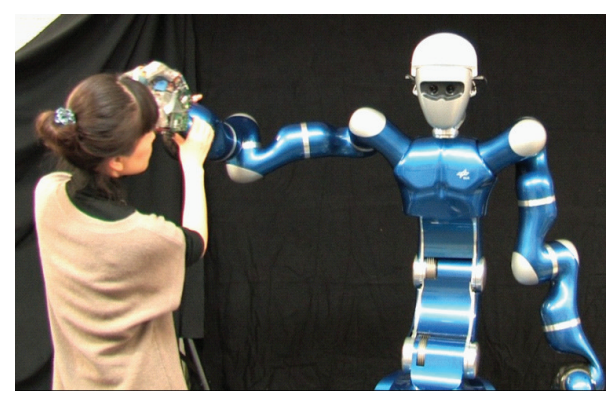

Fig. 1 physical coaching

transfer of skills to the robot, where the user can demonstrate or refine the task in the robot's environment while feeling its capabilities and limitations. With the new development of compliant controllers, backdrivable motors and artificial skins, new perspectives in learning arose by exploiting the natural teaching propensity of the user, already being familiar with social interaction such as scaffolding or kinesthetic teaching . In most works, kinesthetic teaching was realized by deactivating individual selected joints (e.g. by setting very low servo gains) or by a gravity compensation controller. As a consequence, these approaches often lead to unsynchronized motions because the teacher moves motors one by one rather than demonstrating natural coordinated movements. These limitations can be overcome by combining imitation of human's whole body motion with a compliant behavior for physical interaction.

Kinesthetic teaching of a legged humanoid robot has been hardly studied. In existing works, an interaction control approach for the upper body motion was combined with a lower body balancing algorithm based on the reaction null space approach, however, in order to keep ZMP stable upper body might make big momentum and no disturbance estimation was used to take external force into account for balancing explicitly. In practice, disturbance estimation can contribute greatly to different situations, like legged humanoid kinesthetic teaching and incremental kinesthetic teaching of multiple tasks based on the priority control.

\section{Proposed Approach}

In order to achieve intuitive teaching of natural motions, we proposed a method for incremental learning by using physical interaction [1] [3] [4]. In order to ensure synchronization of complex whole body motions on a humanoid robot, our imitation learning procedure starts with observation learning (i.e. whole body motion retargetting from a human demonstrator to a robot) prior to 


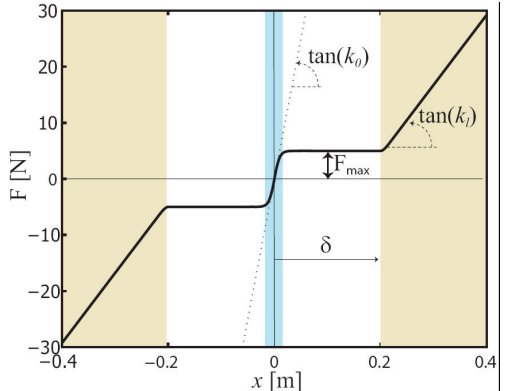

Fig. 2 Nonlinear stiffness in the compliance controller.

kinesthetic motion refinements. There are a wide range of research works on motion retargetting either kinematics level or dynamics level. During the iterative kinesthetic teaching, in which a human supervisor physically interacts with the robot, the user can correct undesired aspects of the retargetted motion resulting from kinematic differences and mapping errors. Considering the physical motion refinement strategy, we can identify the following demands on the real-time controller.

- Trajectory tracking in a free motion phase (where there is no physical interaction).

- Allow a compliant behavior for physical guidance (so that the robot can be distracted from the nominal trajectory by a human teacher).

- Allow to limit the range of allowed deviation from the nominal trajectory (in order to avoid that the human accidentally disturbs the robot motion in an undesired way during the physical coaching).

In order to fulfill these aforementioned requirements, a customized nonlinear impedance control is proposed as shown in Fig. 2. The proposed impedance controller allows to combine tracking of motion primitives in freespace with a kinesthetic modification by a human supervisor.

We extended the kinesthetic teaching based on disturbance observers using force torque sensors in two aspects: to a legged robot [5], and to teaching multiple tasks using task transition [4]. In the legged full body humanoid case [5], the challenge is how to ensure balancing even there are external forces exerted by a human. If the robot is too stiff, a small teaching force can make the robot tilt over. On the other hand if robot is too complaint, a robot is pushed too much and cannot be recover any more neither. For detection of external forces we apply a momentum based disturbance observer. These external forces are used as a trigger for implementing a compliant behavior at the interaction point and are integrated into a predictive balancing algorithm, which predicts the effect of external forces on balancing.

In the second case [4], the focus is how to teach and iteratively refine multiple tasks (end-effector and null pace tasks) only by kinesthetic teaching. Although a whole body motion of a high DOF system is difficult to teach only by the kinesthetic teaching, if we utilize the interaction force measurements well, the robot can be taught to make use of all its degrees-of-freedom (DoF) without any external visual perception (eg., camera for observ- ing human motions). In order to achieve this goal we applied a multi-priority kinematic controller, called Task Transition Control (TTC), in a framework of kinesthetic teaching. On the real-time level, end-effector and nullspace motion primitives, as well as the physical guidance are considered as prioritized tasks. The transitions among these tasks and their insertion and removal are managed by the TTC according to the specified transition parameters. This allows to introduce a customized task which guarantees a proper and smooth response to the applied external forces during the kinesthetic teaching.

\section{Conclusion}

This extended abstract showed our recent work in the field of programming by demonstration and physical human robot interaction. As a baseline, an imitation method for commanding humanoid whole body motion was shown. Then, a refinement process of learned motion primitives using kinesthetic teaching was proposed. By combining both human motion retargetting and kinesthetic demonstrations, a robot can learn synchronized and coordinated high dimensional movements with a nonlinear impedance controller. Kinesthetic teaching of a legged humanoid robot is realized by a predictive balancing algorithm applying a momentum based disturbance observer. Another extension have been made for multiple task learning only based on kinesthetic teaching based on a priority control scheme which allows continuous transition of priority changes. The proposed methods were implemented and evaluated using a wheeled and a legged humanoid robot and a 7 DOF light weight robot.

As an ongoing work, we develop an incremental kinesthetic teaching method of stable dynamical systems using Gaussian process regression. The approach can modify the dynamics of a generic autonomous dynamical systems incrementally every time a new demonstration is provided. A control input is learned from demonstrations to locally modify the trajectory of the system while preserving the stability properties of the reshaped DS.

\section{References}

[1] D. Lee and C. Ott, "Incremental motion primitive learning by physical coaching using impedance control," IEEE/RSJ Int. Conf. on Intelligent Robots and Systems (IROS), 2010.

[2] D. Kulic, C. Ott, D. Lee, and Y. Nakamura, "Incremental Learning of Full Body Motion Primitives through Human Motion Observation," The International Journal of Robotics Research (IJRR), 2012.

[3] D. Lee, C. Ott, "Incremental Kinesthetic Teaching of Motion Primitives Using the Motion Refinement Tube," Autonomous Robots, 2011.

[4] M. Saveriano, S. An, and D. Lee, “Incremental kinesthetic teaching of end-effector and null-space motion primitives," IEEE International Conference on Robotics and Automation (ICRA), pp. 1025-1031, 2015

[5] C. Ott, B. Henz and D. Lee "Kinesthetic teaching of humanoid motion based on whole-body compliance control with interaction-aware balancing," IEEE/RSJ Int. Conf. on Intelligent Robots and Systems (IROS), pp. 4615-4621, 2013.

[6] D. Lee, C. Ott and Y. Nakamura, "Mimetic Communication with Impedance Control for Physical Human-Robot Interaction," IEEE International Conference on Robotics and Automation (ICRA), pp. 1535-1542, 2009 